\title{
The movement of amino acids into rabbit oviductal fluid
}

\author{
H. J. Leese, Sue Aldridge and Kate S. Jeffries \\ Department of Biology, University of York, York YO1 5DD, U.K.
}

\begin{abstract}
Summary. Twenty-three amino acids appeared linearly in oviduct fluid to reach an average concentration $27 \%$ of that in the plasma, with glycine and alanine present in greatest amount. In contrast, the concentration of urea in the fluid approached its plasma concentration after $1 \mathrm{~h}$, and remained at this level for the duration of the experiment. The oviduct was unable to bring about the overall uphill transport of amino acids from plasma to oviduct lumen. The amounts of amino acids appearing in the oviduct lumen of rabbits injected 3 days previously with hCG were mostly similar to those of the controls, but the movements of 6 neutral amino acids appeared to be enhanced relative to their plasma concentrations following ovulation.
\end{abstract}

\section{Introduction}

Measurements of free amino acids in rabbit oviductal fluid have been reported by Gregoire, Gongsakdi \& Rakoff (1961), Hamner \& Williams (1965), Engle et al. (1968) and Iritani, Nishikawa, Gomes \& VanDemark (1971). The most detailed of these studies is that by Iritani et al. (1971) who found a total of 19 free amino acids in oviductal fluid collected daily by cannulation. We have now followed the appearance of amino acids in saline medium recirculated through the lumen of intact rabbit oviducts, for the relatively short period of $4 \mathrm{~h}$, in an attempt to discover more about the mechanism of this transport process.

\section{Materials and Methods}

The apparatus and experimental procedure were identical to those described by Leese \& Aldridge (1979). The perfusion medium was $0.9 \%(\mathrm{w} / \mathrm{v}) \mathrm{NaCl}$ and $2 \mathrm{ml}$ were recirculated through the cannulated oviduct lumen of anaesthetized rabbits at a rate of $50 \mu \mathrm{l} / \mathrm{min}$. After $4 \mathrm{~h}$, samples of the perfusion medium and plasma were deproteinized and neutralized. The extracts were then re-acidified to a $\mathrm{pH}$ of $2 \cdot 2-2.7$ by the addition of a small quantity of concentrated $\mathrm{HCl}$, and placed in an amino acid autoanalyser (Jeol 6AH) using lithium citrate buffers. Tryptophan is very sensitive to degradation by acid and no attempt was made to analyse the peaks given by this amino acid. In some experiments, ovulation was induced by i.v. injection of 100 i.u. hCG (Pregnyl: Organon Laboratories Ltd, Morden, Surrey) and the rabbits were used 3 days later.

\section{Results}

After $4 \mathrm{~h}, 23$ amino acids had appeared in the fluid perfusing the oviduct lumen (Table 1). A further 4 amino acids were detected, and tentatively identified as phosphoethanolamine, 1-methyl histidine, 3-methyl histidine and hydroxy lysine, but no attempt was made to quantify their appearance. Urea was present in large amounts. Glycine and alanine were the amino acids present in greatest quantities. 
Table 1. The appearance of amino acids in fluid after $4 \mathrm{~h}$ circulation in vivo through the oviducts of untreated and hCG-treated rabbits (see text)

\begin{tabular}{|c|c|c|c|c|}
\hline & \multicolumn{2}{|c|}{ Untreated } & \multicolumn{2}{|c|}{ hCG-treated } \\
\hline & $\begin{array}{l}\text { Amount appearing } \\
(\mathrm{nmol} / \mathrm{h})\end{array}$ & $\begin{array}{l}\text { Fluid : plasma } \\
\text { ratio }\end{array}$ & $\begin{array}{c}\text { Amount appearing } \\
(\mathrm{nmol} / \mathrm{h})\end{array}$ & $\begin{array}{l}\text { Fluid : plasma } \\
\text { ratio }\end{array}$ \\
\hline Phosphoserine & $3.65 \pm 0.7(12)$ & $0.23 \pm 0.04(11)$ & N.D. & N.D. \\
\hline Taurine & $9.15 \pm 1.2(16)$ & $0.29 \pm 0.05(13)$ & $4 \pm 0.5$ & $0.31 \pm 0.06(6)$ \\
\hline Urea & $7155 \pm 1230(11)$ & $1.00 \pm 0.15(12)$ & N.D. & N.D. \\
\hline Aspartic acid & $2.9 \pm 0.4(12)$ & $0.29 \pm 0.05(6)$ & $2.6 \pm 0.5(7)$ & $0.20 \pm 0.05(7)$ \\
\hline Threonine & $7.7 \pm 0.6(11)$ & $0.23 \pm 0.03(9)$ & $9.5 \pm 1.8(7)$ & $0.24 \pm 0.04(5)$ \\
\hline Serine & $20.3 \pm 1.8(12)$ & $0.23 \pm 0.02(7)$ & $20.5 \pm 5.5(7)$ & $0.29 \pm 0.03(4)$ \\
\hline Asparagine & $23 \cdot 3 \pm 7.6(8)$ & $0.14 \pm 0.03(4)$ & N.D. & N.D. \\
\hline Glutamic acid & $14 \cdot 2 \pm 2 \cdot 2(15)$ & $0.27 \pm 0.04(12)$ & $10 \pm 2 \cdot 4$ & $0.44 \pm 0.14(5)$ \\
\hline Glutamine & $30.3 \pm 3.7(12)$ & $0.22 \pm 0.04(10)$ & $16.5 \pm 3.5(7)^{*}$ & $0.27(3)$ \\
\hline Proline & $7.8 \pm 0.5(6)$ & $0.12 \pm 0.01(4)$ & $10 \pm 1.8(5)$ & $0.19 \pm 0.05(5)$ \\
\hline Glycine & $324 \pm 40(16)$ & $0.53 \pm 0.06(14)$ & $250 \pm 60(7)$ & $0.89 \pm 0.22(7)$ \\
\hline Alanine & $63 \pm 9.1(16)$ & $0.29 \pm 0.03(12)$ & $51 \pm 10.5(7)$ & $0.63 \pm 0.19(7)^{*}$ \\
\hline Valine & $24 \cdot 3 \pm 2.9(16)$ & $0.16 \pm 0.01(11)$ & $30 \cdot 5 \pm 7(7)$ & $0.33 \pm 0.06(7)^{*}$ \\
\hline Cysteine & $2.1 \pm 0.3(6)$ & $0.06 \pm 0.02(4)$ & $4 \cdot 1 \pm 1 \cdot 8(4)$ & $0.14(2)$ \\
\hline Methionine & $14 \pm 2 \cdot 6(10)$ & $0.35 \pm 0.04(6)$ & $5.0 \pm 0.9(5)^{*}$ & $0.20(3)$ \\
\hline Cystathione & $6.2 \pm 0.6(12)$ & $0.27 \pm 0.05(11)$ & N.D. & N.D. \\
\hline Isoleucine & $12.9 \pm 1.0(16)$ & $0.21 \pm 0.02(12)$ & $15 \cdot 5 \pm 2 \cdot 1(7)$ & $0.39 \pm 0.07(7)^{*}$ \\
\hline Leucine & $30 \cdot 5 \pm 3 \cdot 2(16)$ & $0.29 \pm 0.02(14)$ & $33 \pm 3.6(7)$ & $0.54 \pm 0.07(7)^{*}$ \\
\hline Tyrosine & $11.9 \pm 1.2(16)$ & $0.26 \pm 0.02(12)$ & $11 \pm 1.9(7)$ & $0.36 \pm 0.05(6)^{*}$ \\
\hline Phenylalanine & $16.3 \pm 1.9(16)$ & $0.29 \pm 0.03$ (14) & $17 \pm 1 \cdot 1(7)$ & $0.61 \pm 0.08(7)^{*}$ \\
\hline Ornithine & $3.6 \pm 0.6(11)$ & $0.18 \pm 0.04(9)$ & $2.2 \pm 0.6(6)$ & $0.16+0.05(6)$ \\
\hline Lysine & $10 \cdot 2 \pm 1 \cdot 2(16)$ & $0.14 \pm 0.02(11)$ & $7.5 \pm 1.9(7)$ & $0.14 \pm 0.04(7)$ \\
\hline Histidine & $9.9 \pm 1.7(13)$ & $0.15 \pm 0.02(12)$ & $5.0 \pm 0.9(7)^{*}$ & $0.23 \pm 0.06(7)$ \\
\hline Arginine & $11.6 \pm 2.5(10)$ & $0.17 \pm 0.03(9)$ & $5.0 \pm 0.8(7)^{*}$ & $0.11 \pm 0.03(7)$ \\
\hline
\end{tabular}

Values are mean \pm s.e.m. with the number of determinations in parentheses. N.D. $=$ not determined.

* Indicates significantly different $(P<0.05$ by Student's $t$ test) from the corresponding untreated value.

Since it is likely that the free amino acids appearing in oviductal fluid originate mainly in the plasma, the final concentration of each amino acid in the fluid was divided by its concentration in the plasma (Table 1). With an average concentration ratio of unity, urea appeared to be in equilibrium with the plasma, and glycine was the amino acid transported to the greatest extent relative to its plasma concentration.

In view of these results, it was considered necessary to establish whether the amino acids were appearing linearly in the perfusion fluid, or whether their rates of transport were beginning to decline during the experimental period. The amino acid content of the fluid perfusing the oviduct was therefore measured after 1 and $2 \mathrm{~h}$, and the results are given in Table 2. Cysteine, proline, methionine and cystathione were not present at 1 and $2 \mathrm{~h}$ in sufficient quantity to be measured accurately. The results indicated that, with the exception of urea, the measurement of the amino acids at $4 \mathrm{~h}$ represented a reliable estimate of their initial rates of appearance in the oviductal fluid. To test whether active transport of amino acids could take place into the rabbit oviductal fluid, a mixture of 21 amino acids, at concentrations similar to those in the plasma, was added to the fluid perfusing two oviducts at the start of incubation, and the final amino acid concentrations were measured after $4 \mathrm{~h}$. In no case did an amino acid concentration in the fluid increase; the mean \pm s.e.m. concentrations were $73 \pm 3.1 \%$ and $79 \pm 3.7 \%$ of their original value, suggesting that the rabbit oviduct cannot bring about the overall uphill transport of amino acids from plasma to oviduct lumen.

The effect of hCG treatment on amino acid appearance is shown in Table 1. With the exception of glutamine, methionine, histidine and arginine, whose appearances were significantly reduced following ovulation, amino acid levels after $4 \mathrm{~h}$ perfusion were similar to those of the 
controls. However, the final fluid/plasma concentration ratios were noticeably higher for many of the amino acids, particularly the neutral ones, alanine, valine, isoleucine, leucine, tyrosine and phenylalanine, and these differences were statistically significant. With the exception of aspartic acid and threonine, all the plasma amino acid concentrations were depressed after hCG treatment (data not shown), and it is reasonable to conclude that the neutral amino acids mentioned above must have been moving into the medium perfusing the oviduct faster, relative to their plasma concentration, to account for their total appearance remaining unchanged after ovulation.

Table 2. The time course of appearance of amino acids in fluid circulating through the rabbit oviduct in vivo, relative to their concentration in the plasma

\begin{tabular}{lccc}
\hline & & Time $(\mathrm{h})$ & \\
\cline { 2 - 4 } & 1 & 2 & 4 \\
\hline Phosphoserine & $\mathrm{N} . \mathrm{D}$ & $0.14 \pm 0.03(5)$ & $0.23 \pm 0.04(11)$ \\
Taurine & $0.11 \pm 0.01(4)$ & $0.16(2)$ & $0.29 \pm 0.05(13)$ \\
Urea & $0.78 \pm 0.09(4)$ & $0.82 \pm 0.03(5)$ & $1.00 \pm 0.15(12)$ \\
Aspartic acid & $0.12 \pm 0.01(4)$ & $0.19 \pm 0.04(5)$ & $0.29 \pm 0.05(6)$ \\
Threonine & $0.05 \pm 0.01(4)$ & $0.10 \pm 0.01(5)$ & $0.23 \pm 0.03(9)$ \\
Serine & $0.06 \pm 0.02(4)$ & $0.10 \pm 0.01(5)$ & $0.23 \pm 0.02(7)$ \\
Asparagine & $\mathrm{N} . \mathrm{D}$. & $0.07 \pm 0.02(5)$ & $0.14 \pm 0.03(4)$ \\
Glutamic acid & $0.10 \pm 0.03$ & $0.14 \pm 0.04(5)$ & $0.27 \pm 0.04(12)$ \\
Glutamine & $0.04(2)$ & $0.07(2)$ & $0.22 \pm 0.04(10)$ \\
Glycine & $0.13 \pm 0.03(4)$ & $0.24 \pm 0.02(5)$ & $0.53 \pm 0.06(14)$ \\
Alanine & $0.08 \pm 0.02(4)$ & $0.13 \pm 0.01(5)$ & $0.29 \pm 0.03(12)$ \\
Valine & $0.06 \pm 0.01(4)$ & $0.10 \pm 0.02(5)$ & $0.16 \pm 0.01(11)$ \\
Isoleucine & $0.07 \pm 0.02(4)$ & $0.13 \pm 0.01(5)$ & $0.21 \pm 0.02(12)$ \\
Leucine & $0.15 \pm 0.01(4)$ & $0.23 \pm 0.02(5)$ & $0.29 \pm 0.02(14)$ \\
Tyrosine & $0.11 \pm 0.03(4)$ & $0.17 \pm 0.10(5)$ & $0.26 \pm 0.02(12)$ \\
Phenylalanine & $0.09 \pm 0.02(4)$ & $0.16 \pm 0.03(5)$ & $0.29 \pm 0.03(14)$ \\
Ornithine & $0.04 \pm 0.01(4)$ & $0.08 \pm 0.01(5)$ & $0.18 \pm 0.04(9)$ \\
Lysine & $0.05 \pm 0.01(4)$ & $0.09 \pm 0.01(5)$ & $0.14 \pm 0.02(11)$ \\
Histidine & $0.05 \pm 0.01(4)$ & $0.08 \pm 0.01(5)$ & $0.15 \pm 0.02(12)$ \\
Arginine & $0.06 \pm 0.02(4)$ & $0.10 \pm 0.02(5)$ & $0.17 \pm 0.03(9)$ \\
\hline
\end{tabular}

Values are mean \pm s.e.m. with the number of determinations in parentheses. N.D. = not determined.

\section{Discussion}

We have shown that the movement of amino acids into the rabbit oviduct lumen may readily be followed over the relatively short period of $4 \mathrm{~h}$. After this time $2.6 \mu \mathrm{mol}$ of amino acids had appeared in the perfusion fluid at an average concentration $27 \%$ of that in the plasma. Apart from urea, the amino acid present in highest quantity was glycine $(1.29 \mu \mathrm{mol})$ and that in lowest amount, cysteine $(0.0084 \mu \mathrm{mol})$, in agreement with the work of Iritani et al. (1971), and representing a 150 -fold difference in quantity. The amino acids with the highest and lowest fluid to plasma concentration ratios were again glycine and cysteine $(0.534$ and 0.057 respectively) but in this case the ratios only differed by a factor of 9 . This suggested that the amino acids were to a considerable extent derived from the plasma, but gave no information on the proportion from this source. As the concentration of amino acids decreased in the lumen when their initial concentrations were close to those of the plasma, they cannot be in simple equilibrium with the plasma. The exception to this generalization is urea the concentration of which in the medium perfusing the oviducts quickly approached its concentration in the plasma. Urea is a small molecule that rapidly crosses cell membranes, and might be expected to be in equilibrium across the oviduct. 
We failed to detect the active transport of amino acids into the medium perfusing the oviduct lumen of oestrous rabbits. In contrast, Jaszczak \& Hafez (1972) reported that the majority of the free amino acids in rabbit uterine fluid $168 \mathrm{~h}$ post coitum were present in higher concentration than in the blastocoelic fluid or in the blood serum. In oviducts from rabbits 3 days after hCG injection, the fluid/plasma concentration ratios were significantly enhanced for 6 amino acids and it is possible that the postovulatory oviduct might be able to effect uphill amino acid transport.

We thank Mr P.G. Humpherson for expert technical assistance, and the Medical Research Council for a research grant.

\section{References}

Engle, C.C., Dunn, J.S., Hood, N.O., Williams, D.J., Foley, C.W. \& Trout, H.J. (1968) Amino acids in sow and rabbit oviduct fluids. J. Anim. Sci. 27, 1786, Abstr.

Gregoire, A.T., Gongsakdi, D. \& Rakoff, A.E. (1961) The free amino acid content of the female rabbit genital tract. Fert. Steril 12, 322-327.

Hamner, C.E. \& Williams, W.L. (1965) Composition of rabbit oviduct secretions. Fert. Steril. 16, 170-176.

Iritani, A., Nishikawa, Y., Gomes, W.R. \& VanDe- mark, N.L. (1971) Secretion rates and chemical composition of oviduct and uterine fluids in rabbits. J. Anim. Sci. 33, 829-835.

Jaszczak, S. \& Hafez, E.S.E. (1972) Free amino acids in uterine and blastocoelic fluids in the rabbit as affected by ovarian steroids. Int. J. Fertil. 17, 191196.

Leese, H.J. \& Aldridge, S. (1979) The movement of pyruvate, lactate and lactate dehydrogenase into rabbit oviductal fluid. J. Reprod. Fert. 56, 619-622.

Received 1 December 1978 